\title{
Transcriptional coactivator PGC-1 $\alpha$ integrates the mammalian clock and energy metabolism
}

\author{
Chang Liu ${ }^{1}$, Siming $\mathrm{Li}^{1}$, Tiecheng $\mathrm{Liu}^{2}$, Jimo Borjigin ${ }^{2} \&$ Jiandie D. Lin ${ }^{1}$
}

The mammalian clock regulates major aspects of energy metabolism, including glucose and lipid homeostasis and mitochondrial oxidative metabolism ${ }^{1,2}$. The biochemical basis for coordinated control of the circadian clock and diverse metabolic pathways is not well understood. Here we show that PGC-1a (Ppargcla), a transcriptional coactivator that regulates energy metabolism ${ }^{3-9}$, is rhythmically expressed in the liver and skeletal muscle of mice. PGC-1 $\alpha$ stimulates the expression of clock genes, notably Bmal1 (Arntl) and Rev-erbo (Nr1d1), through coactivation of the ROR family of orphan nuclear receptors. Mice lacking PGC-1 $\alpha$ show abnormal diurnal rhythms of activity, body temperature and metabolic rate. The disruption of physiological rhythms in these animals is correlated with aberrant expression of clock genes and those involved in energy metabolism. Analyses of PGC-1 $\alpha$ deficient fibroblasts and mice with liver-specific knockdown of PGC-1 $\alpha$ indicate that it is required for cell-autonomous clock function. We have thus identified PGC-1 $\alpha$ as a key component of the circadian oscillator that integrates the mammalian clock and energy metabolism.

Most living organisms exhibit behavioural and physiological rhythms, including activity, sleep, metabolism and body temperature. These rhythms are controlled by a circadian clock and are subject to regulation by light-dark cycles. In mammals, the central and peripheral clocks are controlled by a common transcriptional circuitry that generates rhythmic patterns of gene expression, most notably those encoding metabolic enzymes ${ }^{10-14}$. Perturbations of circadian rhythms have been associated with an increased risk for metabolic disorders in humans ${ }^{15}$. Remarkably, mice with defective clock function develop obesity and have impaired glucose homeostasis ${ }^{16,17}$, suggesting that the regulation of circadian clocks is linked to the pathways of energy metabolism and potentially to the pathogenesis of metabolic diseases. PGC- $1 \alpha$ is an inducible transcriptional coactivator that regulates adaptive energy metabolism in multiple tissues ${ }^{4,6}$. The critical role of this factor in linking the environment to metabolism prompted us to examine whether PGC- $1 \alpha$ regulates the circadian clock and serves as a link between the clock and energy metabolism.

The clock oscillator consists of transcriptional activators and repressors that are assembled into feedback loops ${ }^{18,19}$. To determine the effects of PGC- $1 \alpha$ on clock genes, we transduced primary hepatocytes and $\mathrm{C} 2 \mathrm{C} 12$ myotubes with recombinant adenoviruses expressing green fluorescent protein (GFP) or PGC-1 $\alpha$. Quantitative PCR (qPCR) analyses indicate that PGC- $1 \alpha$ induces the expression of Bmal1 and Clock in both cell types (Fig. 1a). Consistent with messenger RNA expression, Bmall and Clock protein levels are elevated in hepatocytes in response to PGC- $1 \alpha$ (Fig. 1b). Clock and Bmal1 are two basic helix-loop-helix transcription factors that activate the transcription of period (Per) and cryptochrome (Cry) genes ${ }^{18,19}$. Per and Cry proteins in turn inhibit their own expression by repressing Clock/Bmal1 activity, forming the critical feedback loop within the clock circuitry. Interestingly, the effects of PGC- $1 \alpha$ on Per and Cry gene expression seem to be cell-type-specific. Previous studies have implicated orphan nuclear receptors of the ROR and Rev-erb families in the control of circadian clock function ${ }^{13,20-22}$. Although PGC- $1 \alpha$ exerts only modest effects on the expression of ROR $\alpha$ (Rora) and $R O R \gamma$ (Rorc), it significantly induces Rev-erb $\alpha$ and $R e v-e r b \beta$ expression in both cell types (Fig. 1a).

Recent expression profiling studies have identified a large number of genes that show rhythmic expression in the liver ${ }^{1-13}$. To assess the role of PGC- $1 \alpha$ in the regulation of circadian gene expression at the transcriptome level, we performed microarray analysis on total RNA isolated from primary hepatocytes transduced with GFP or PGC-1 $\alpha$ adenoviruses. We integrated the PGC- $1 \alpha$ microarray data set with a previously described set of 335 oscillating hepatic genes ${ }^{11}$ to determine global effects of PGC- $1 \alpha$ on circadian genes. As shown in Fig. 1c, 47 of these circadian-regulated genes are induced by PGC- $1 \alpha$ by more than 1.8-fold, with the expression of many clustering at the onset and during early dark phase (Supplementary Table 1). Analysis of PGC$1 \alpha$ mRNA levels in mouse liver revealed a diurnal rhythm that peaks at CT13 (CT0 is the onset at hour 0 of subjective light period), gradually declines thereafter, and reaches a nadir at CT1 (Fig. 1d). PGC- $1 \alpha$ expression is similarly rhythmic in skeletal muscle, though to a lesser degree. The expression of other PGC-1 coactivators, that is, $P G C-1 \beta$ and $P R C$, also show circadian rhythms in these tissues. Immunoblotting analyses indicate that PGC- $1 \alpha$ protein reaches peak levels in early dark phase (Fig. 1e), coinciding with the activation of Bmal1 transcription and its mRNA accumulation. Interestingly, the short isoform of PGC- $1 \beta$ shows a diurnal rhythm that peaks several hours before PGC-1 $\alpha$. In contrast, the protein levels of Brm (also known as Smarca2), a component of the SWI/SNF chromatinremodelling complexes, remain unchanged. Taken together, these data indicate that rhythmic activation of PGC- $1 \alpha$, and possibly other PGC-1 coactivators, may have an important role in the regulation of clock function.

To identify transcription factors that mediate the regulation of clock genes by PGC- $1 \alpha$, we examined the ability of PGC- $1 \alpha$ to synergize with these factors in the regulation of Bmall transcription. PGC$1 \alpha$ dramatically augments the transcriptional activity of $\operatorname{ROR} \alpha$ and $\mathrm{ROR} \gamma$, but not $\mathrm{C} / \mathrm{EBP} \alpha$, on a Bmal1 promoter reporter (Fig. 2a and Supplementary Fig. 1). Coactivation of ROR $\alpha$ by PGC- $1 \alpha$ was previously observed for genes involved in muscle lipid metabolism ${ }^{23}$. In contrast, PGC- $1 \alpha$ exerts modest effects on the transcriptional activity of Clock/Bmal1 (data not shown). The synergistic effects of ROR and PGC- $1 \alpha$ are abolished when the ROR-binding sites (RORE) on the proximal Bmal1 promoter are mutated. Physical interaction assays indicate that PGC- $1 \alpha$ binds to ROR $\alpha$ and ROR $\gamma$ through the LXXLL motifs (Supplementary Fig. 1). This functional synergy between 
a
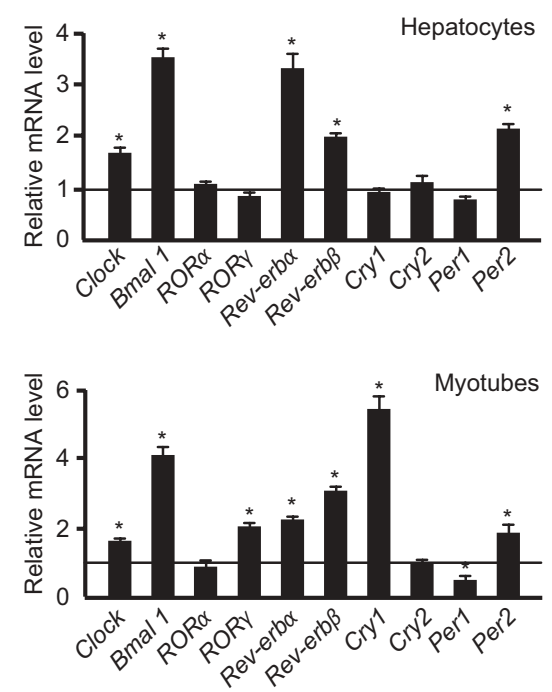

b

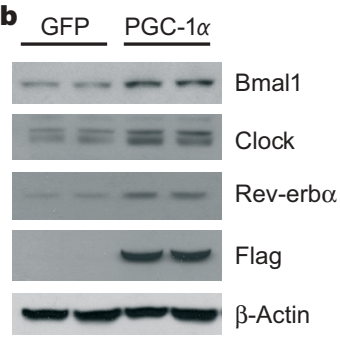

e

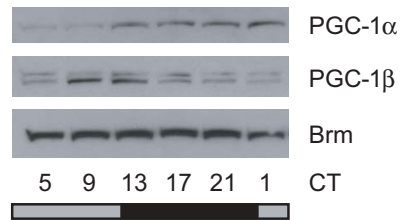

c

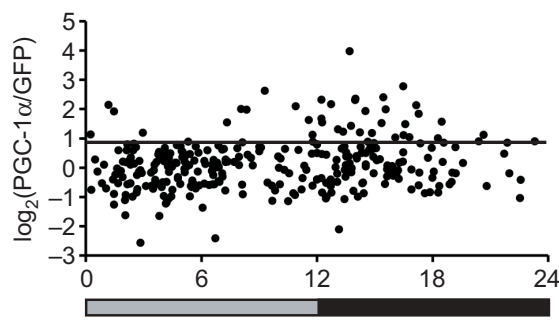

$\mathrm{CT}(\mathrm{h})$
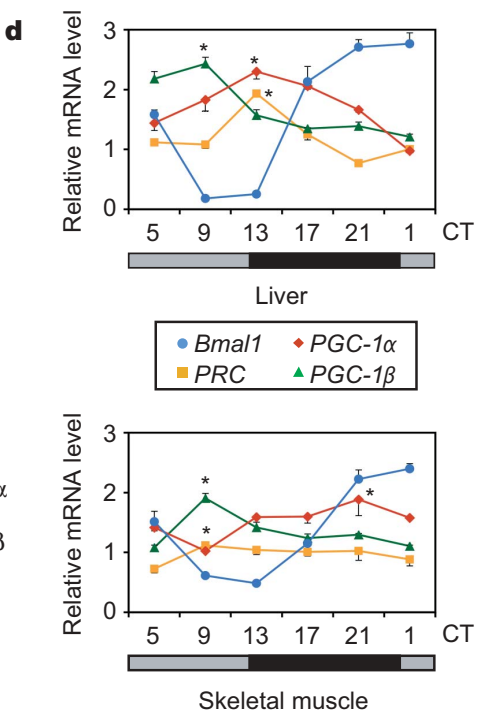

Figure 1 Regulation of clock genes by PGC-1a. a, qPCR analysis of clock genes in primary hepatocytes and $\mathrm{C} 2 \mathrm{C} 12$ myotubes. Shown is fold-induction by PGC- $1 \alpha$. Data represent mean \pm s.d. ${ }^{\star} P<0.02$, PGC- $1 \alpha$ versus GFP. b, Immunoblots of hepatocyte lysates using the indicated antibodies. c, Effects of PGC- $1 \alpha$ on circadian-regulated genes in the liver. Fold induction by

a
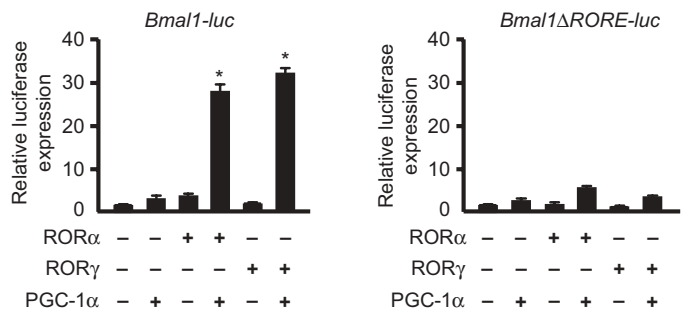

b

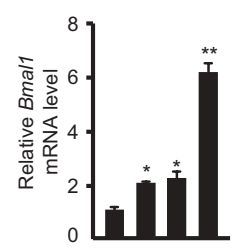

c
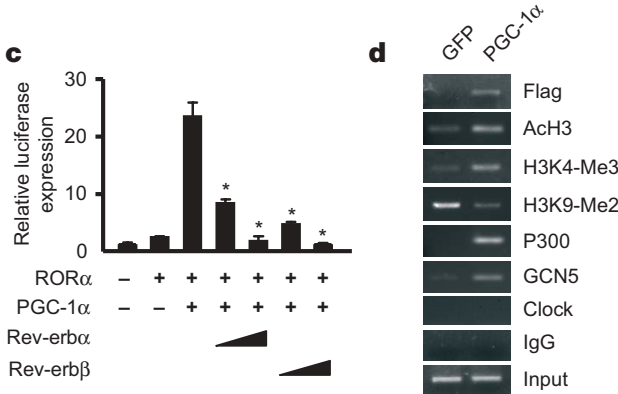

Figure 2 Regulation of Bmal1 gene transcription by PGC-1 $\alpha$ and ROR. a, Reporter gene assays using wild-type (Bmal1-luc) or RORE-mutant (Bmal14RORE-luc) Bmal1-luciferase reporters. ${ }^{\star} P<0.01, P G C-1 \alpha$ versus control. b, qPCR analysis of Bmall expression in transduced hepatocytes. a, b, Error bars, s.d. ${ }^{\star} P<0.05$, ROR $\alpha$ or PGC- $1 \alpha$ versus Control; ${ }^{\star \star} P<0.01$, ROR $\alpha$ plus PGC- $1 \alpha$ versus ROR $\alpha$. c, Reporter gene assays using Bmal1-luc in the presence of Rev-erb $\alpha$ or Rev-erb $\beta$. Shown with mean \pm s.d. ${ }^{\star} P<0.001$, Rev-erb versus control. d, ChIP assay with the indicated antibodies using HepG2 cells transduced with GFP or Flag-PGC-1 $\alpha$ adenoviruses. PCR primers amplify a fragment flanking the proximal RORE.
PGC- $1 \alpha$ of the circadian gene set was calculated on the basis of microarray data, log-transformed and plotted against the respective peak timepoints of expression in the liver (solid line, 1.8 -fold). d, qPCR analysis of liver and skeletal muscle gene expression. Data represent mean \pm s.d. ${ }^{\star} P<0.01$, peak versus nadir. e, Immunoblots of liver nuclear extracts using the indicated antibodies.

ROR $\alpha$ and PGC- $1 \alpha$ is also observed for the endogenous Bmal1 gene (Fig. 2b). Previous studies indicate that Rev-erb $\alpha$ negatively regulates Bmal1 transcription by recruiting corepressor proteins ${ }^{22}$, indicating that the induction of Rev-erb factors may provide a negative feedback mechanism for PGC- $1 \alpha$ function. Consistent with this, both Rev$\operatorname{erb} \alpha$ and Rev-erb $\beta$ drastically repress the stimulatory effects of PGC-1 $\alpha$ on the Bmal1-luc reporter (Fig. 2c). These results illustrate that the ability of PGC- $1 \alpha$ to activate Bmall transcription is modulated by the relative abundance of the ROR and Rev-erb families of orphan receptors.

Chromatin immunoprecipitation (ChIP) assays in HepG2 cells indicate that PGC- $1 \alpha$ is present near RORE on the proximal Bmal1 promoter (Fig. 2d). In addition, PGC- $1 \alpha$ recruits histone acetyltransferases, including p300 and GCN5, but not Clock, a recently described DNA-binding histone acetyltransferase ${ }^{24}$, to this site. Histone hyperacetylation is associated with transcriptional activation. On the other hand, trimethylation of lysine 4 of histone 3 (H3K4me3) is a hallmark for actively transcribed genes, whereas H3K9 dimethylation ( $\mathrm{H} 3 \mathrm{~K} 9 \mathrm{me} 2)$ is typically found in heterochromatin and silenced genes $^{25}$. Remarkably, PGC-1 $\alpha$ expression leads to a robust increase in histone $\mathrm{H} 3$ acetylation and $\mathrm{H} 3 \mathrm{~K} 4 \mathrm{me} 3$ levels with a corresponding reduction in $\mathrm{H} 3 \mathrm{~K} 9 \mathrm{me} 2$ levels on the proximal Bmal1 promoter. These results indicate that PGC- $1 \alpha$ activates Bmall transcription by altering the local chromatin environment from a repressive to an active state.

The ability of PGC- $1 \alpha$ to regulate the expression of clock genes indicates that this factor may impact on the circadian clock, thereby linking clock oscillators to energy metabolism. To test whether PGC$1 \alpha$ is required for clock function in vivo, we monitored locomotor activity and body temperature in wild-type and $P G C-1 \alpha$ null mice 
using telemetry. Wild-type mice exhibit a free-running period of approximately $23.7 \mathrm{~h}$ in constant darkness, whereas the free-running period is approximately $24.0 \mathrm{~h}$ in PGC- $1 \alpha$ null mice (Fig. 3a). In addition, locomotor activity is evenly distributed throughout the subjective night in the null mice. These results indicate that PGC$1 \alpha$ regulates locomotor behaviour in mice and is required for maintaining normal circadian periods. Diurnal oscillation of body temperature is also distinct between these two genotypes (Fig. 3b). Because PGC- $1 \alpha$ is an important regulator of energy metabolism, we next examined whether the daily oscillation of metabolic rate is altered in the null mice. Using indirect calorimetry, we measured oxygen consumption rate $\left(\mathrm{VO}_{2}\right)$ in wild-type and $P G C-1 \alpha$ null mice over a period of three days ${ }^{6}$. Consistent with locomotor activity and body temperature rhythms, $\mathrm{VO}_{2}$ is significantly higher in the dark phase (Fig. 3c). Compared with wild-type mice, $P G C$ - $1 \alpha$ null mice have elevated $\mathrm{VO}_{2}$ in both light and dark phases, though the diurnal oscillation of $\mathrm{VO}_{2}$ is severely blunted. These results indicate that PGC- $1 \alpha$ is a critical component of the clock circuitry that may link the clock oscillator to metabolism.

Analysis of clock gene expression indicates that the rise of Bmall mRNA levels is significantly impaired (37\% lower at CT19), although the rhythmic expression of Bmall is nearly intact in $P G C-1 \alpha$ null livers (Fig. 3d). In contrast, peak Bmal1 mRNA level is higher in PGC-1 $\alpha$-deficient skeletal muscle (Fig. 3e), indicating that PGC- $1 \alpha$ deficiency differentially perturbs clock genes in different tissues. Diurnal rhythms of Clock and Per1 mRNA are also disrupted in the liver and skeletal muscle of $P G C$ - $1 \alpha$ null mice, respectively. We next examine whether PGC- $1 \alpha$ is required for the cyclic expression of various enzymes involved in energy metabolism. PEPCK (PCK1), a rate-limiting enzyme of hepatic gluconeogenesis, shows a daily cycle that peaks at CT13 in the wild-type liver. PGC- $1 \alpha$ seems to be dispensable for the rhythmic expression of this enzyme. Nevertheless, the mRNA levels of PEPCK are constitutively elevated at CT1 and CT13 (Fig. 3d), in a similar way to the previous studies ${ }^{6}$. Pyruvate dehydrogenase kinase 4 (PDK4) is a key regulator of the pyruvate dehydrogenase complex that controls the flux of pyruvate into the tricarboxylic acid cycle. The diurnal PDK4 expression is severely impaired in PGC-1 $\alpha$-deficient liver and skeletal muscle, although the phase of PDK4 oscillation seems to be preserved. Similarly, the cyclic expression of fatty acid binding protein $3(F a b p 3 / H-F A B P)$ as well as other mitochondrial genes involved in oxidative phosphorylation (OXPHOS), such as aconitase (Aco2) and Cox4a (Cox4i1), is also disrupted in $P G C-1 \alpha$ null skeletal muscle. Together, our results illustrate that PGC- $1 \alpha$ is essential for oscillatory expression of clock and metabolic genes in vivo.

The defects in circadian rhythms of clock and metabolic gene expression in $P G C-1 \alpha$ null mice could potentially be affected by altered central clock function. Previous work indicates that peripheral oscillators can be reset by restricted feeding, essentially uncoupling them from the master pacemaker in the suprachiasmatic nucleus ${ }^{26}$. To determine whether PGC- $1 \alpha$ is required for phase resetting of peripheral clocks in response to feeding, we subjected wildtype and null mice to night feeding and then switched them to day feeding. Blood glucose concentrations show robust oscillation when the mice were fed exclusively at night (Fig. 4a). The phase of glucose oscillation is shifted by approximately $12 \mathrm{~h}$ after the feeding switch in both groups. However, the amplitude of blood glucose rhythms is significantly dampened in mice lacking PGC-1 $\alpha$. qPCR analyses revealed that the expression of $P G C-1 \alpha$ and clock genes are reversed in response to restricted feeding in wild-type livers (Fig. 4b). In contrast, feeding-induced resetting of clock gene expression is significantly disrupted in PGC-1 $\alpha$ null liver and skeletal muscle (Fig. 4b and Supplementary Fig. 2). The expression of Bmall in the suprachiasmatic nucleus is also reduced in $P G C-1 \alpha$ null mice (Supplementary Fig. 2); however, the feeding switch does not seem to alter Bmal1 expression patterns. Interestingly, $P G C-1 \beta$ mRNA levels are elevated at some timepoints in these tissues.

To rule out the possibility that the perturbation of peripheral clocks in $P G C-1 \alpha$ null mice may be secondary to a compromised central clock, we examined cell-autonomous clock function in vivo a
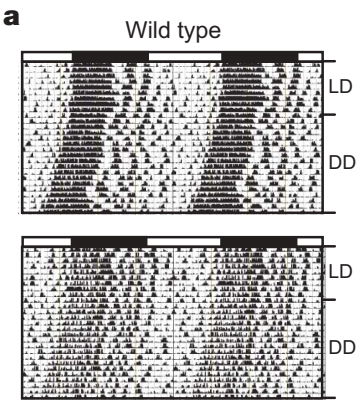

b
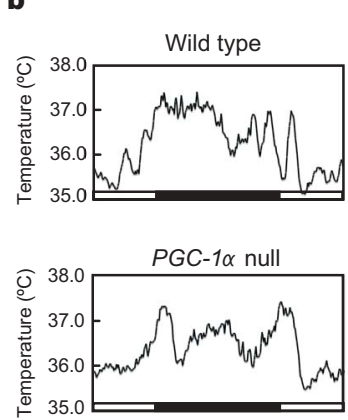
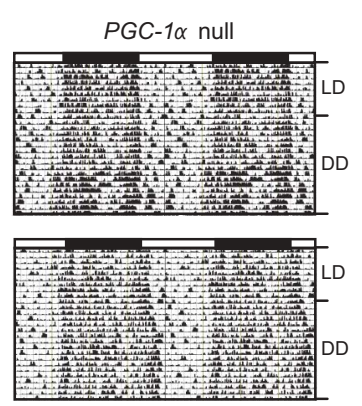

c
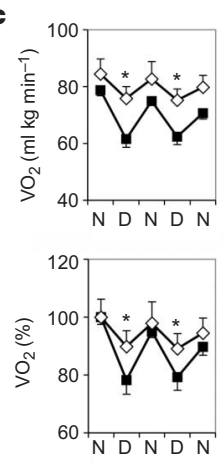

e
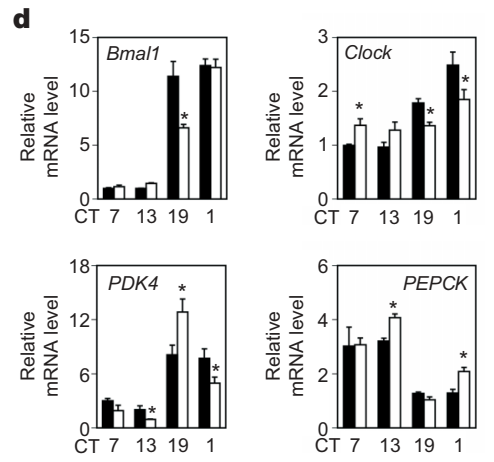
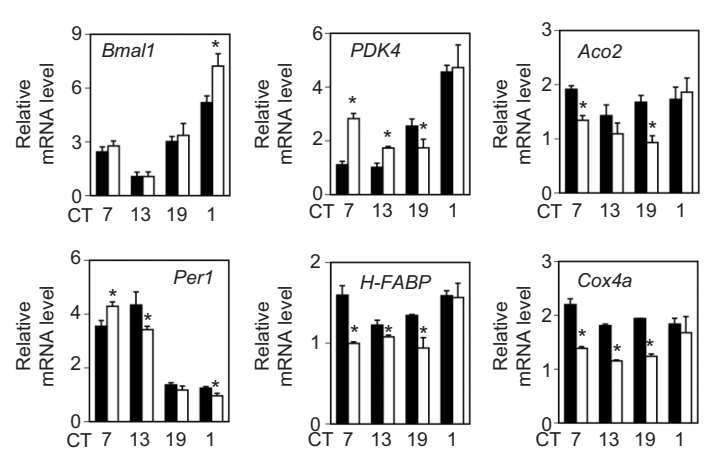

Figure 3 | Disruption of clock function in PGC-1 $\alpha$ null mice. a, Locomotor activity in wild-type and $P G C-1 \alpha$ null mice under normal light:dark cycles 12:12 h (LD) or constant darkness (DD). Data were plotted in duplicate columns in each panel. $\mathbf{b}$, Representative traces of core body temperature in wild-type and $P G C-1 \alpha$ null mice. c, Metabolic rate in wild-type (filled square, $n=5$ ) and $P G C-1 \alpha$ null (open diamond, $n=6$ ) mice. Shown are average and normalized $\mathrm{VO}_{2}$ during subjective night $(\mathrm{N})$ and day $(\mathrm{D})$. Error bars, s.e.m. d, e, qPCR analysis of gene expression in pooled liver (d) and skeletal muscle (e). Error bars, s.d. of the mean. ${ }^{*} P<0.05$, wild-type (filled box) versus null mice (open box). 
a
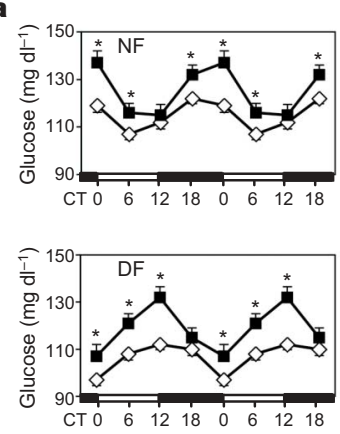

c

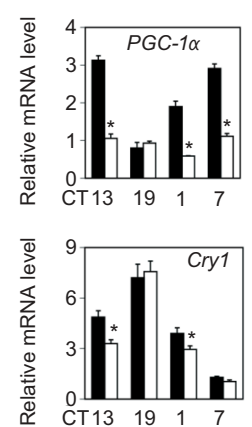

b
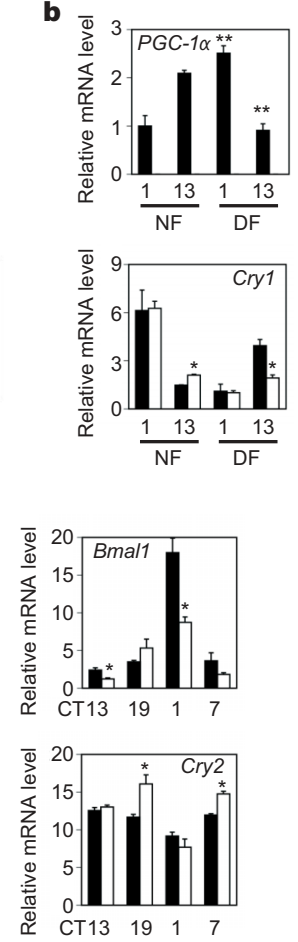

Figure 4 | Cell-autonomous role of PGC-1 $\alpha$ in clock regulation. a, Plasma glucose concentrations in wild-type (filled square, $n=16$ ) and $P G C-1 \alpha$ null mice (open diamond, $n=14$ ) subjected to night $(\mathrm{NF})$ or day (DF) feeding. Data were plotted in duplicate columns. b, qPCR analysis of total liver RNA isolated from wild-type (filled box) and PGC-1 $\alpha$ null (open box) mice. c, qPCR analyses of hepatic gene expression in mice transduced with control (filled box) or PGC-1 $\alpha$ RNAi (open box) adenoviruses. Error bars in

as well as in cultured cells. C57/Bl6J mice were transduced through the tail vein with adenoviruses expressing random or short hairpin RNA (shRNA) directed towards PGC-1 $1 \alpha^{27}$. RNA interference (RNAi) knockdown of $P G C-1 \alpha$ in the liver significantly disrupts rhythmic expression of Bmal1, Cry1 and Cry2 in this tissue, but not in the suprachiasmatic nucleus (Fig. 4c and Supplementary Fig. 2). Serum shock has been demonstrated to induce rhythmic clock gene expression in cultured fibroblasts ${ }^{28}$. We exposed immortalized brown preadipocytes to brief serum shock and analysed expression of clock genes over a period of $36 \mathrm{~h}$. Serum shock leads to robust oscillation of Bmall and Rev-erb $\alpha$ expression in wild-type cells (Fig. 4d). In contrast, rhythmic expression of these genes is essentially abolished in cells lacking PGC- $1 \alpha$. Consistent with these results, the ability of ROR $\alpha$ to induce the expression of Bmall and Rev-erb $\alpha$ is impaired in hepatocytes lacking PGC- $1 \alpha$ (Supplementary Fig. 3), indicating that PGC- $1 \alpha$ is required for the transcriptional activity of ROR $\alpha$ in this context. We conclude from these studies that PGC- $1 \alpha$ exerts its effects on the circadian clock largely in a tissue- and cell-autonomous manner.

We have identified PGC- $1 \alpha$, a major metabolic regulator, as a critical component of the mammalian clock. PGC- $1 \alpha$ stimulates the expression of Bmall through coactivating the ROR family of orphan nuclear receptors and is essential for normal circadian rhythms. Because PGC- $1 \alpha$ expression is highly responsive to nutritional signals and potentially light, our findings support a mechanism through which energy metabolism and circadian clock can be directly coupled at the transcriptional level (Fig. 4e). PGC-1 $\alpha$ null mice are resistant to diet-induced obesity and are more insulinsensitive ${ }^{6,17}$, whereas Clock mutant mice develop obesity. These differences are probably due to the fact that $P G C-1 \alpha$ null mice are hyperactive and have a higher metabolic rate in the absence of increased food intake. Our findings raise an intriguing possibility that the expression and/or activity of PGC- $1 \alpha$ itself may be regulated
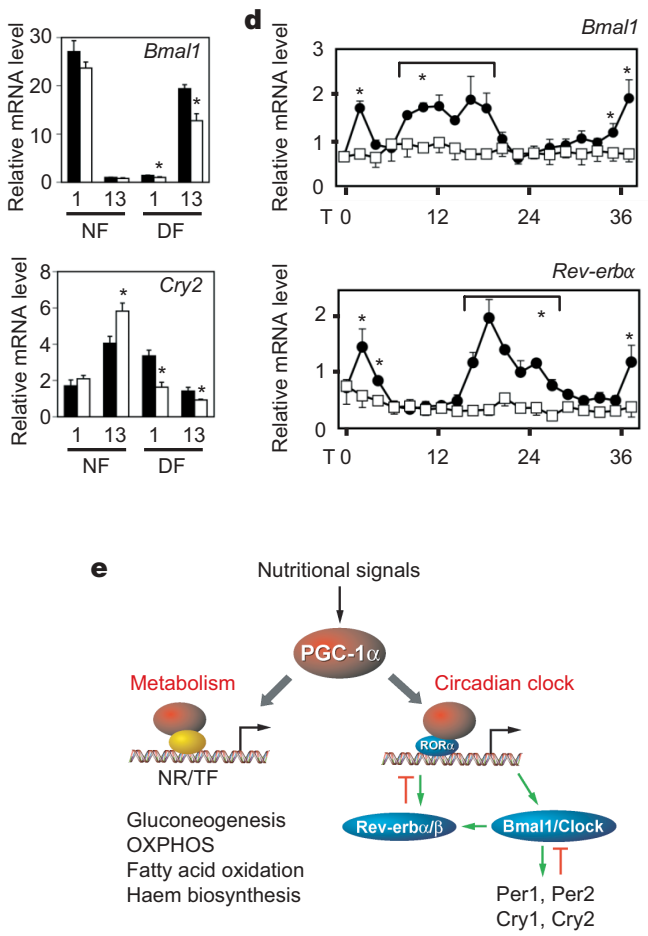

a-c indicate s.e.m. a, b, c, ${ }^{\star} P<0.05$ wild-type versus null mice; ${ }^{\star \star} P<0.01$ NF versus DF. d, Time course expression of clock genes in wild-type (filled circle) and $P G C-1 \alpha$ null (open square) fibroblasts following serum shock. Error bars, s.d. ${ }^{\star} P<0.05$ wild-type versus null cells. e, Model for coordinated regulation of circadian clock and energy metabolism through PGC-1 $\alpha$.

by components of the clock oscillator. Because feeding and locomotor activity are regulated by circadian clocks, it is possible that rhythmic PGC- $1 \alpha$ expression is controlled by these physiological and behavioural rhythms. Alternatively, PGC- $1 \alpha$ is associated with the SirT1 histone deacetylase complex ${ }^{29}$ and may directly sense the metabolic state of the cell, in a similar way to the regulation of the clock homologue NPAS2 by redox status ${ }^{30}$. Disruption of circadian rhythms has been implicated in the pathogenesis of metabolic disorders. Our studies have uncovered a potential molecular target that could simultaneously modulate circadian clocks and energy metabolism.

\section{METHODS SUMMARY}

Transcriptional analyses. Adenoviral transduction, reporter gene assays, and ChIP experiments were performed as previously described ${ }^{6,9}$. For gene expression analyses, total RNA was isolated from tissues or cultured cells using Trizol reagents (Invitrogen), reversed transcribed and analysed by quantitative PCR using SYBR-Green.

Animal experiments. For activity and body temperature monitoring, wild-type and PGC-1 $\alpha$ null mice were implanted with a G2 Minimiter II probe in the abdominal cavity (MiniMitter) and allowed to recover for two weeks prior to the initiation of monitoring using telemetric receivers. Voluntary activity and body temperature were recorded under light:dark cycles 12:12 h for two weeks before switching to constant darkness for three more weeks. For restricted feeding, male wild-type and $P G C-1 \alpha$ null mice were fed exclusively at night for ten days. Blood glucose levels were measured at the end of night feeding as well as four days following the switch to day feeding. Tissues were harvested at CT1 and 13 for both feeding groups for qPCR analysis of gene expression. For liverspecific PGC-1 $\alpha$ knockdown, wild-type C57/Bl6J male mice were administered control or PGC-1 $\alpha$ RNAi adenoviruses via the tail vein. Tissues were harvested from transduced animals five days after adenoviral transduction.

Serum shock. Immortalized fibroblasts from wild-type and $P G C-1 \alpha$ null mice were established and maintained in DMEM supplemented with 10\% FBS. For serum shock, media of confluent cultures was replaced with DMEM plus 50\% horse serum $(t=0)$ and, after 1 hour, the cells were washed once with PBS and 
incubated with serum-free DMEM. Total RNA was extracted at the indicated time points and processed for $\mathrm{qPCR}$ analysis. More details are provided in Methods.

Full Methods and any associated references are available in the online version of the paper at www.nature.com/nature.

\section{Received 5 January; accepted 21 March 2007.}

Published online 2 May 2007.

1. Rutter, J., Reick, M. \& McKnight, S. L. Metabolism and the control of circadian rhythms. Annu. Rev. Biochem. 71, 307-331 (2002).

2. Schibler, U. \& Naef, F. Cellular oscillators: rhythmic gene expression and metabolism. Curr. Opin. Cell Biol. 17, 223-229 (2005).

3. Kelly, D. P. \& Scarpulla, R. C. Transcriptional regulatory circuits controlling mitochondrial biogenesis and function. Genes Dev. 18, 357-368 (2004).

4. Leone, T. C. et al. PGC-1 $\alpha$ deficiency causes multi-system energy metabolic derangements: muscle dysfunction, abnormal weight control and hepatic steatosis. PLoS Biol. 3, e101 (2005).

5. Lin, J., Handschin, C. \& Spiegelman, B. M. Metabolic control through the PGC-1 family of transcription coactivators. Cell Metab. 1, 361-370 (2005).

6. Lin, J. et al. Defects in adaptive energy metabolism with CNS-linked hyperactivity in PGC-1 $\alpha$ null mice. Cell 119, 121-135 (2004).

7. Puigserver, P. et al. A cold-inducible coactivator of nuclear receptors linked to adaptive thermogenesis. Cell 92, 829-839 (1998).

8. $\mathrm{Wu}$, Z. et al. Mechanisms controlling mitochondrial biogenesis and respiration through the thermogenic coactivator PGC-1. Cell 98, 115-124 (1999).

9. Yoon, J. C. et al. Control of hepatic gluconeogenesis through the transcriptional coactivator PGC-1. Nature 413, 131-138 (2001)

10. Lowrey, P. L. \& Takahashi, J. S. Mammalian circadian biology: elucidating genomewide levels of temporal organization. Annu. Rev. Genomics Hum. Genet. 5, 407-441 (2004).

11. Panda, S. et al. Coordinated transcription of key pathways in the mouse by the circadian clock. Cell 109, 307-320 (2002).

12. Storch, K. F. et al. Extensive and divergent circadian gene expression in liver and heart. Nature 417, 78-83 (2002).

13. Ueda, H. R. et al. A transcription factor response element for gene expression during circadian night. Nature 418, 534-539 (2002).

14. Wijnen, H. \& Young, M. W. Interplay of circadian clocks and metabolic rhythms. Annu. Rev. Genet. 40, 409-448 (2006).

15. Karlsson, B., Knutsson, A. \& Lindahl, B. Is there an association between shift work and having a metabolic syndrome? Results from a population based study of 27,485 people. Occup. Environ. Med. 58, 747-752 (2001).

16. Rudic, R. D. et al. BMAL1 and CLOCK, two essential components of the circadian clock, are involved in glucose homeostasis. PLoS Biol. 2, e377 (2004).

17. Turek, F. W. et al. Obesity and metabolic syndrome in circadian Clock mutant mice. Science 308, 1043-1045 (2005).
18. Schibler, U. \& Sassone-Corsi, P. A web of circadian pacemakers. Cell 111, 919-922 (2002).

19. Reppert, S. M. \& Weaver, D. R. Coordination of circadian timing in mammals. Nature 418, 935-941 (2002)

20. Preitner, N. et al. The orphan nuclear receptor REV-ERB $\alpha$ controls circadian transcription within the positive limb of the mammalian circadian oscillator. Cell 110, 251-260 (2002)

21. Sato, T. K. et al. A functional genomics strategy reveals Rora as a component of the mammalian circadian clock. Neuron 43, 527-537 (2004).

22. Yin, L., Wang, J., Klein, P. S. \& Lazar, M. A. Nuclear receptor Rev-erba is a critical lithium-sensitive component of the circadian clock. Science 311, 1002-1005 (2006).

23. Lau, P., Nixon, S. J., Parton, R. G. \& Muscat, G. E. ROR $\alpha$ regulates the expression of genes involved in lipid homeostasis in skeletal muscle cells: caveolin-3 and CPT-1 are direct targets of ROR. J. Biol. Chem. 279, 36828-36840 (2004).

24. Doi, M., Hirayama, J. \& Sassone-Corsi, P. Circadian regulator CLOCK is a histone acetyltransferase. Cell 125, 497-508 (2006)

25. Martin, C. \& Zhang, Y. The diverse functions of histone lysine methylation. Nature Rev. Mol. Cell Biol. 6, 838-849 (2005).

26. Damiola, F. et al. Restricted feeding uncouples circadian oscillators in peripheral tissues from the central pacemaker in the suprachiasmatic nucleus. Genes Dev. 14, 2950-2961 (2000).

27. Koo, S. H. et al. PGC-1 promotes insulin resistance in liver through PPAR- $\alpha-$ dependent induction of TRB-3. Nature Med. 10, 530-534 (2004).

28. Balsalobre, A., Damiola, F. \& Schibler, U. A serum shock induces circadian gene expression in mammalian tissue culture cells. Cell 93, 929-937 (1998).

29. Rodgers, J. T. et al. Nutrient control of glucose homeostasis through a complex of PGC-1 $\alpha$ and SIRT1. Nature 434, 113-118 (2005).

30. Rutter, J., Reick, M., Wu, L. C. \& McKnight, S. L. Regulation of clock and NPAS2 DNA binding by the redox state of NAD cofactors. Science 293, 510-514 (2001).

Supplementary Information is linked to the online version of the paper at www.nature.com/nature.

Acknowledgements We thank J. Hogenesch and T. Sato for Bmal1-luc, ROR $\alpha$ and Rev-erb plasmids; S. Reppert for the E-box luciferase constructs; J. Takahashi for the Bmal1 and Clock expression plasmids; and M. Montminy for PGC-1 $1 \alpha$ RNA adenovirus. We also thank A. Saltiel and B. Spiegelman for comments on the manuscript and P.-H. Wu for discussions. This work is supported by NIDDK (J.D.L.) and the University of Michigan BSSP program (J.D.L.).

Author Contributions C.L., S.L., J.B. and J.D.L. designed the research. C.L., S.L., T.L., and J.D.L. performed the experiments. C.L., S.L., T.L., J.B. and J.D.L. analysed the data. J.D.L. wrote the paper.

Author Information Reprints and permissions information is available at www.nature.com/reprints. The authors declare no competing financial interests. Correspondence and requests for materials should be addressed to J.D.L. (jdlin@umich.edu). 


\section{METHODS}

Adenoviral transduction. The maintenance and adenoviral transduction of primary hepatocytes and $\mathrm{C} 2 \mathrm{C} 12$ myotubes was performed, as previously described $^{6}$. Total RNA was isolated using Trizol reagents (Invitrogen) $48 \mathrm{~h}$ following transduction, reverse transcribed, and analysed by qPCR using SYBR Green. A complete list of PCR primers is shown in Supplementary Table 2. Primers for ribosomal protein 36B4 were included for normalization. Microarray analysis was performed on total RNA isolated from hepatocytes transduced with GFP or PGC-1 $\alpha$ adenoviruses using U74av2 chips (Affymetrix).

Animals. All animal procedures were approved by the University Committee on Use and Care of Animals. For analysis of PGC-1 $\alpha$ expression in tissues, male C57/Bl6J mice of 12 weeks of age were kept under light:dark (LD) 12:12 h and subsequently subjected to constant darkness for $36 \mathrm{~h}$. Tissues from five mice were dissected every $4 \mathrm{~h}$ for a total of $24 \mathrm{~h}$ thereafter. Total RNA was isolated and analysed by qPCR using gene-specific primers. For analysis of gene expression in wild-type and $P G C-1 \alpha$ null mice, three to five mice of each genotype were euthanized every six hours. Tissues were immediately frozen and subsequently processed for qPCR analysis and immunoblotting analyses.

For activity and body temperature monitoring, 3-month old wild-type and PGC- $1 \alpha$ null mice were implanted with a G2 Minimiter II probe in abdominal cavity (MiniMitter). Animals were allowed to recover for two weeks prior to the initiation of monitoring using telemetric receivers. Voluntary activity and body temperature were recorded under LD 12:12 h for two weeks before switching to constant darkness for three more weeks. Data were analysed using software supplied by the manufacturer. For restricted feeding, male wild-type and PGC-1 $\alpha$ null mice were fed exclusively at night for ten days. Blood glucose levels were measured at the end of night feeding as well as four days following the switch to day feeding. Tissues were harvested at CT1 and 13 for both feeding groups for qPCR analysis of gene expression. For liver-specific PGC- $1 \alpha$ knockdown, a total of $32 \mathrm{C} 57 / \mathrm{Bl} 6 \mathrm{~J}$ male mice were administered control or PGC-1 $\alpha$ RNAi adenoviruses ( 0.1 absorbance units per mouse), as previously described. Five days following tail vein injection, tissues were harvested from transduced animals at CT1, 7, 13 and 19 (four mice per treatment).

Reporter gene assays. Reporter gene assays were performed in BOSC 293 cells, as previously described. In a typical experiment, $25 \mathrm{ng}$ of reporter plasmids were mixed with $10 \mathrm{ng}$ of expression constructs for transcription factors in the presence or absence of PGC- $1 \alpha$ expression construct. Equal amounts of DNA were used for all transfection combinations by adding appropriate vector DNA. Relative luciferase activities were determined $48 \mathrm{~h}$ following transfection. All transfection experiments were performed in triplicates.

Serum shock. Immortalized fibroblasts from wild-type and PGC- $1 \alpha$ null mice were established and maintained in DMEM supplemented with 10\% FBS. For serum shock, media of confluent cultures was replaced with DMEM plus 50\% horse serum $(t=0)$, and after $1 \mathrm{~h}$, the cells were washed once with PBS and incubated with serum-free DMEM. Total RNA was extracted at the indicated time points and processed for qPCR analysis using 36B4 as a normalization control.

ChIP assay. Chromatin immunoprecipitation was performed essentially as described by the Upstate Biotechnology (www.upstate.com). Briefly, HepG2 hepatoma cells were transduced with GFP or Flag-PGC-1 $\alpha$ adenoviruses for $48 \mathrm{~h}$. Chromatin lysates were prepared, pre-cleared with Protein-A agarose beads, and immunoprecipitated with antibodies against Flag (Sigma), K9dimethylated and K4-trimethylated histone $\mathrm{H} 3$ (Abcam), acetylated histone H3 (Upstate Biotechnology), p300 and Clock (Santa Cruz Biotech), GCN5 (BioLegend), or normal mouse IgG (Sigma) in the presence of BSA and salmon sperm DNA. Beads were extensively washed before reverse cross-linking. DNA was purified using a PCR purification kit (Qiagen) and subsequently analysed by PCR using primers flanking the proximal RORE on the human Bmall promoter, as detailed in Supplementary Table 2. 